\title{
Synchronous and Metachronous Metastatic Breast Cancer, with Different Histology and Opposite Immunophenotype, Treated with Combination of Chemotherapy, Anti-Her2, and Endocrine Therapy: A Case Report
}

\author{
Giacomina Megaro $^{a} \quad$ Luigi Rossi $^{\mathrm{b}}$ Serena Ceddia ${ }^{\mathrm{b}}$ Marsela Sinjari ${ }^{\mathrm{b}}$ \\ Adele Mannino $^{b} \quad$ Elisa Gozzi ${ }^{b}$ Antonella Cosimatib Martina Brandi ${ }^{b}$ \\ Victoria Bitca $^{b} \quad$ Ilaria Toscanic $^{c}$ Giuseppe Cimino $^{d}$ Silverio Tomao ${ }^{\mathrm{e}}$ \\ aDepartment of Paediatric Haematology and Oncology, Ospedale Bambin Gesù, \\ Rome, Italy; 'UOC of Oncology - ASL Latina - Distretto 1, University of Rome "Sapienza", \\ Aprilia, Italy; 'Medical Oncology Ospedale G. da Saliceto, Piacenza, Italy; ${ }^{\mathrm{d} D e p a r t m e n t}$ \\ of Cellular Biotechnology and Haematology, University of Rome "Sapienza", Rome, Italy; \\ eMedical Oncology Unit A Policlinico Umberto I, University of Rome "Sapienza", Rome, Italy
}

\section{Keywords}

Bilateral breast cancer - Contralateral breast - Infiltrating ductal carcinoma - Infiltrating lobular carcinoma - Metachronous cancer - Synchronous cancer - Case report

\section{Abstract}

In the case of our patient, the synergic action of endocrine therapy and chemotherapy plus dual anti-HER2 combination allowed a complete disease control. Therapy should be scheduled by considering the two cancers as individual entities. The approach to breast cancer is changing from being considered a singular disease to a multiform one, according to current research focused on biological markers such as HER2, ERs, and PRs, with important implications in clinical, prognostic, and therapeutic features.

\section{Introduction}

Synchronous bilateral breast cancer is defined as contralateral breast cancer diagnosed within 1 year of previous breast cancer [1].

The incidence of bilateral breast cancer is about $3 \%$ of all breast cancers: the synchronous tumors represent $0.6 \%$, while the metachronous are $2.2 \%$. 
Megaro et al.: Synchronous, Metachronous Metastatic Breast Cancer Treated with Combination of Therapies

Fig. 1. Positron emission tomography before treatment with docetaxeltrastuzumab-pertuzumab.

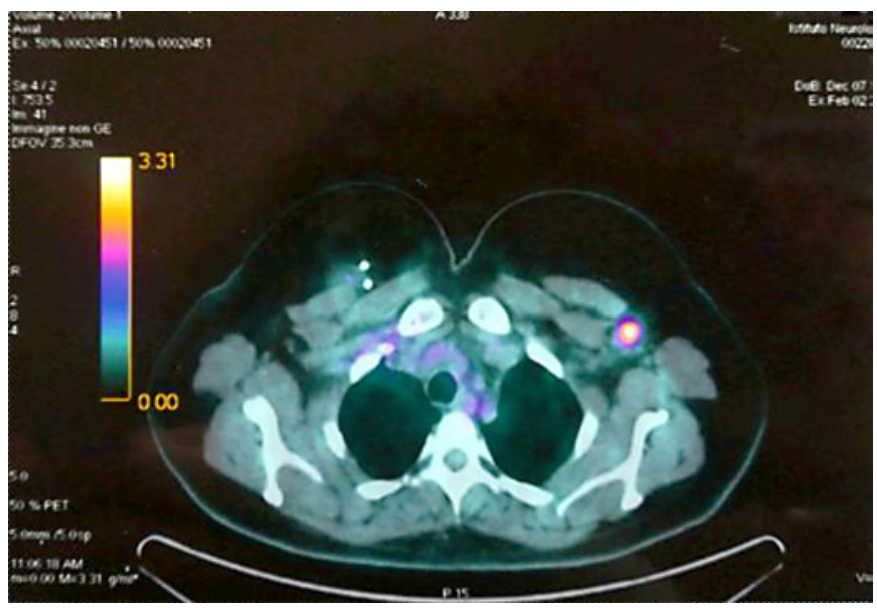

Two primary breast cancers may be a result of a genetic predisposition, exposure to common environmental risk factors, or an accumulation of two unrelated events [2].

In young patients, the relative risk of developing metachronous bilateral breast cancer is high. The risk of developing a contralateral primary breast cancer in patients with a history of previous breast cancer is about 5 times higher as compared to the occurrence of breast cancer in an unaffected individual [3].

Cases of metachronous bilateral breast cancer with different histopathological and molecular structures are rare [4-8].

We will describe the case of a young patient with metastatic synchronous lobular breast cancer and metachronous ductal breast cancer with differing tumor biology.

\section{Case Presentation}

A 49-year-old Caucasian female patient, with an unremarkable medical and breast history, performance status ECOG 0, came to our observation in July 2014 after a lumpectomy for multifocal invasive lobular cancer (ILC) of right breast. Tumor biology was luminal type: estrogen receptor (ER) 85\%, progesterone receptor (PR) 5\%, Ki67 2\%, human epidermal growth factor receptor 2 (HER2) negative. A subsequent bilateral mammary magnetic resonance imaging showed a suspicious mass at the left breast. The core biopsy deployed for infiltrating lobular carcinoma luminal type as the first was removed. Patient performed computerized tomography (CT) scan, which revealed multiple bone metastases. BRCA mutational state was performed and revealed wild type for BRCA1 and BRCA2.

A first-line endocrine therapy (ET) with anastrozole and LH-RH analog was set up. At the same time, patient started bisphosphonates (zoledronic acid $4 \mathrm{mg}$ q28).

A partial response on breast lesion and bone metastases was obtained.

After 2 years from diagnosis of the first cancer, a CT scan showed a new lesion to left breast with progressive growth in the following 6 months, confirmed by ultrasound scan and mammography.

The positron emission tomography (PET) scan confirmed new breast lesion with standardized uptake value of 20 and multiple left axillary and thoracic lymphadenopathy (Fig. 1).

A new biopsy was performed, and it revealed totally different tumor biology: ER- and PR-negative, HER2-positive invasive ductal carcinoma (IDC). Ki67 was 80\%. 
Fig. 2. Positron emission tomography after treatment with docetaxeltrastuzumab-pertuzumab.

Megaro et al.: Synchronous, Metachronous Metastatic Breast Cancer Treated with Combination of Therapies

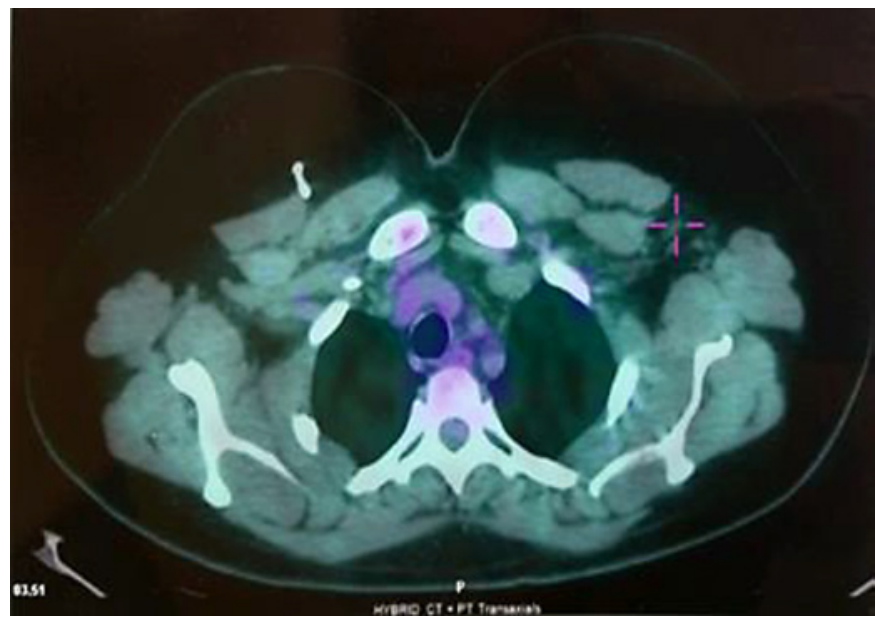

Fig. 3. Positron emission tomography before second-line ET with fulvestrant.

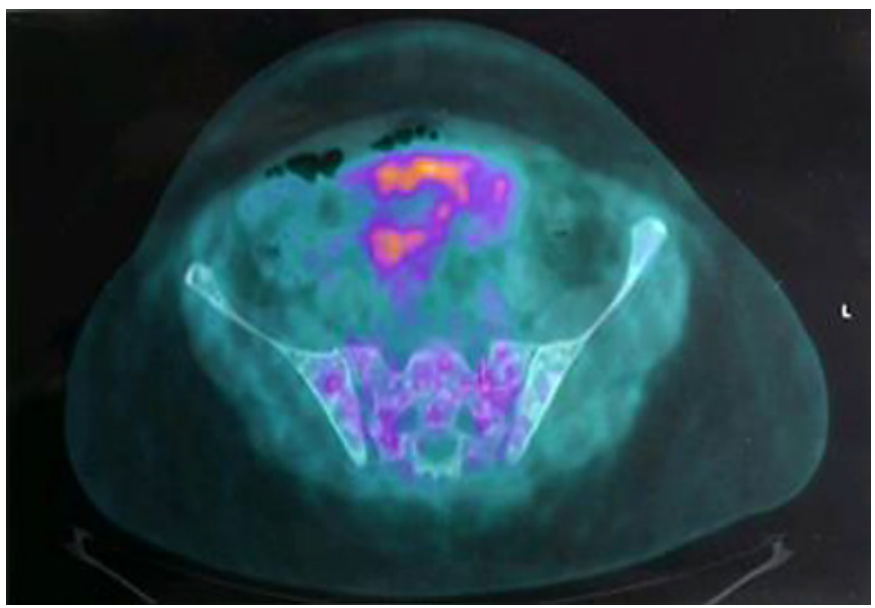

Fig. 4. Positron emission tomography after second-line ET with fulvestrant.

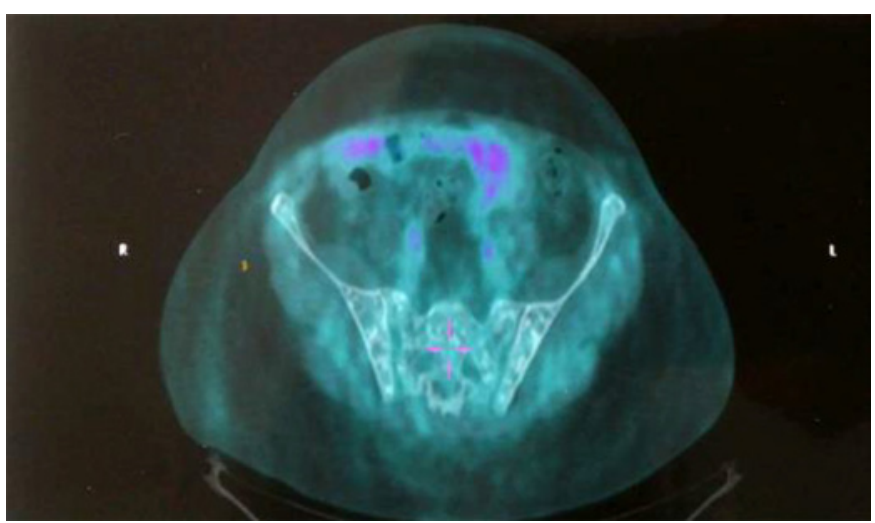

According to the different biology of metachronous breast cancer, patient started treatment with docetaxel-trastuzumab-pertuzumab, in line with CLEOPATRA protocol [9]. Cardiac function was good with a left ventricular ejection fraction of $65 \%$. Patient continued anastrozole.

After 3 months, PET scan showed a partial response to ductal breast lesion and lymphadenopathy but progression of disease on bone metastases (Fig. 2, Fig. 3). Anastrozole was stopped. 
Megaro et al.: Synchronous, Metachronous Metastatic Breast Cancer Treated with Combination of Therapies

Because progression of lobular cancer and response of the ductal cancer, patient started second-line ET with fulvestrant and continued pertuzumab-trastuzumab. No early or late toxicities related to the treatments were observed. The following PET scan attested a reduction of the metabolic activity on the bone lesions (Fig. 4).

After 9 months, PET scan showed a complete metabolic response.

In July 2019, the last PET scan proved an adequate control of cancer with no signs of pathological disease and the CT scan showed no radiological variation.

After 36 months of therapy with trastuzumab-pertuzumab and fulvestrant, patient still maintains complete response to the treatment, does not refer any symptoms, and her left ventricular ejection fraction is still $65 \%$.

\section{Discussion}

Bilateral breast cancer is uncommon with an incidence of 2-5\%. The second tumor can be synchronous or metachronous.

Compared with IDC, ILCs are generally diagnosed at more advanced stage, more frequent node invasion, and more often luminal type [10]. In our patient, bilateral ILC was luminal type diagnosed at metastatic stage, while metachronous IDC was HER2-like with nodal involvement.

Breast cancer is associated with female hormones, environmental and lifestyle factors, and genetic risk factors (BRCA, TP53, CDH1).

Germline mutations in BRCA1 and TP53 are associated with IDC, BRCA2 with both ductal and lobular type. Mutations in $\mathrm{CDH} 1$ are exclusively associated with ILC.

Considering the young age and the bilateral nature of the primary tumor, our patient was nominated for a BRCA study. BRCA status was wild type for BRCA1 and BRCA2.

Our patient is affected by two different breast cancers: the first metastatic bilateral lobular cancer and the second metachronous locally advanced ductal cancer.

The two tumors, moreover, express opposite biological characteristics: the lobular of luminal type $A$ and the ductal of type HER2-like. The receptor status can indicate monoclonal or polyclonal origin in case of bilateral or synchronous breast cancer and can help to personalize treatment.

Approximately $20 \%$ of all breast cancers have gene amplification or overexpression (or both) of HER2 [11], with poorest prognosis because of aggressive phenotype.

Our patient was treated according to CLEOPATRA trial with chemotherapy and dual antiHER2 combination: trastuzumab and pertuzumab [9]. The copresence of luminal A breast cancer was treated with ET [12].

Several studies have provided numerous lines of evidence that HER2 pathways may directly or indirectly contribute to the development of resistance to hormone therapy. Identified mechanisms of resistance [13]:

- Activation of the PI3K/Akt/mTOR and p42/44 MAPK pathways, mediated by HER2,

induces a downregulation of both ER and PgR expression.

- The PAX2 transcriptional factor loss or deregulation seems to be associated with the acquisition of a HER2-driven phenotype by preventing the HER2 transcriptional

repression by estrogen-ER and tamoxifen-ER complexes [14].

In metastatic patients chronically exposed to trastuzumab, tumors with the expression of hormone receptors showed a sudden upregulation of ER followed by a decrease of response to anti-HER2 therapies [15].

The standard clinical practice has been dramatically influenced by the recent CLEOPATRA and EMILIA [16] trials. From the subgroup analysis of the CLEOPATRA study, the coexpression of hormone receptor and HER2 was associated with lower sensitivity to the double anti-HER2 
Megaro et al.: Synchronous, Metachronous Metastatic Breast Cancer Treated with Combination of Therapies

block and lower OS [9]. The limited sensitivity is probably due to crosstalk ER-HER2. None of the two trials tested the novel anti-HER2 therapy combined with hormone therapy; therefore, the current standard of care for metastatic HER2+ breast cancer consists of trastuzumab combined with pertuzumab and a taxane for first line and TDM1 for second line in disease relapse within 6 months of adjuvant therapy.

In the case of our patient, however, the presence of HER2 and hormone receptor on two different tumors, instead of one, has allowed the two treatments to act with greater synergy.

At 38 months from the beginning of this therapeutic strategy, the patient is asymptomatic, with PS ECOG 0 and has a complete metabolic response.

The approach to breast cancer is changing from being considered a singular disease to a multiform one. The importance of biological markers such as HER2, ERs, and PRs, and their prognostic value should be evaluated in the therapeutic choices of this widespread disease.

\section{Statement of Ethics}

The authors declare that ethics approval was not required for this case report. Written informed consent was obtained from the patient for publication of this case and accompanying images. A copy of the written consent is available for review by the Editor-in-Chief of this journal.

\section{Disclosure Statement}

The authors declare no conflict of interest.

\section{Funding Sources}

No funding was received.

\section{Author Contributions}

G.M. conceived the need to describe the case and wrote the manuscript. L.R. was the primary editor of the manuscript. S.C. wrote the abstract. M.S. and E.G. reviewed the literature. A.M. reviewed the manuscript according to the authors' instructions. A.C. and M.B. made the English correction. I.T. prepared Figures 1 and 2. V.B. prepared Figures 3 and 4. G.C. and S.T. coordinated the realization of the manuscript. All authors read and approved the final manuscript.

\section{References}

1 Al-Jurf AS, Jochimsen PR, Urdaneta LF, Scott DH. Factors influencing survival in bilateral breast cancer. J Surg Oncol. 1981;16(4):343-8.

2 Dawson PJ, Maloney T, Gimotty P, Juneau P, Ownby H, Wolman SR. Bilateral breast cancer: one disease or two? Breast Cancer Res Treat. 1991 Nov 1;19(3):233-44.

3 Vaittinen P, Hemminki K. Risk factors and age-incidence relationships for contralateral breast cancer. Int J Cancer. 2000;88(6):998-1002.

4 Dalal AK, Gupta A, Singal R, Dalal U, Attri AK, Jain P, et al. Bilateral breast carcinoma - a rare case report. J Med Life. 2011 Feb 15;4(1):94-6. 
5 Kappikeri VK, Kriplani AM. Bilateral synchronous carcinoma breast - a rare case presentation. Springerplus. 2015;4:193.

6 Barvi G, Banerjee M, Mukherjee S, Das TK. An interesting case of primary bilateral synchronous breast carcinoma with mucinous carcinoma in one. NJLM. 2016;5:7-9

7 Singla A, Kaur N, Walia DS, Singla D. Bilateral metachronous breast carcinoma: a rare case report. Int J Appl Basic Med Res. 2018 Jun;8(2):126-8.

8 Esclovon JW, Ponder M, Aydin N, Misra S. Challenges of treating incidental synchronous bilateral breast cancer with differing tumour biology. BMJ Case Rep. 2016 Aug 18;2016:bcr2016216201.

9 Swain SM, Baselga J, Kim SB, Ro J, Semiglazov V, Campone M, et al. Pertuzumab, trastuzumab, and docetaxel in HER2-positive metastatic breast cancer. N Engl J Med. 2015 Feb 19;372(8):724-34.

10 Li CI, Uribe DJ, Daling JR. Clinical characteristics of different histologic types of breast cancer. Br J Cancer. 2005 Oct; 93(9):1046-52.

11 Wolff AC, Hammond ME, Schwartz JN, Hagerty KL, Allred DC, Cote RJ, et al. American Society of Clinical Oncology/College of American Pathologists guideline recommendations for human epidermal growth factor receptor 2 testing in breast cancer. Arch Pathol Lab Med 2007;131(1):18-43.

12 Tomao F MD, Spinelli G, Vici P, Pisanelli G, Cascialli G, Frati L, et al. Current role and safety profile of aromatase inhibitors in early breast cancer. Expert Rev Anticancer Ther. 2011;11(8):1253-63.

13 Agus DB, Akita RW, Fox WD, Lewis GD, Higgins B, Pisacane PI, et al. Targeting ligand-activated ErbB2 signaling inhibits breast and prostate tumor growth. Cancer Cell. 2002 Aug 1;2(2):127-37.

14 Montemurro F, Di Cosimo S, Arpino G. Human epidermal growth factor receptor 2 (HER2)-positive and hormone receptor-positive breast cancer: new insights into molecular interactions and clinical implications. Ann Oncol. 2013 Nov;24(11):2715-24.

15 Nahta R, O'Regan RM. Therapeutic implications of estrogen receptor signaling in HER2-positive breast cancers. Breast Cancer Res Treat. 2012 Aug;135(1):39-48.

16 Verma S, Miles D, Gianni L, Krop IE, Welslau M, Baselga J, et al. Trastuzumab emtansine for HER2-positive advanced breast cancer. N Engl J Med. 2012 Nov 8;367(19):1783-91. 